\title{
MR-Compatible Servo Drive - First Results
}

\author{
P. P. Pott, A. Hiemstra, H. F. Schlaak
}

Microtechnology and Electromechanical Systems Lab., Institute for Electromechanical Design, TU Darmstadt, Darmstadt, Germany

\section{Introduction}

For medical robotic applications radio translucent and MR-compatible actuators are of great interest. The latter implies the absence of any ferro-electric material and otherwise conductive parts. In the case of magnetic tracking such actuators could also be useful.

The work at hand describes the technological background and the control of a vane motor with reduction gear and optical encoder in a compact package made completely out of synthetic materials.

\section{Methods}

Appropriate materials where chosen after a thorough literature research. Also, an actuation and sensing concept was elaborated based on manufacturer's data, text books, and design methodology. The actuator and angular sensor were built in the institute's machine shop while the reduction gear was provided by a commercial supplier. A model-based controller was implemented on a $\mu \mathrm{C}$. As development environment NI's LabView was used.

\section{Results}

The final system consists of a custom-built optical encoder and a vane motor both made completely out of PVC and PE. As reduction gear a conventional polymeric harmonic drive gear is used. To control the actuator a ball valve and a directional control valve are used. The control loop is closed by an AVR micro controller. The actuator is designed to work with hoses up to $5 \mathrm{~m}$ in length. So, also the optical encoder has optical fibres of this length. The device is able to provide up to $5 \mathrm{Nm}$ of torque at $50 \mathrm{rpm}$ while the optical encoder currently provides an angular resolution of $1^{\circ}$.

\section{Conclusion}

The presented servo-drive provides reasonable torque for many medical applications where the absence of metals and conductive material is desirable. It should also be radio translucent to be used in a CT scanner. However, the actuator does not yet provide reliable performance as the utilised plastics wear out quickly. Nevertheless, in contrast to wellknown pneumatic stepping actuators the presented actuator's controllability and speed are independent of the hose length. 